\title{
Silicon Nanocrystals on the Surface of Standard Si Wafers: A Micro-Raman Investigation
}

\author{
Mohammed S. G. Mohammed ${ }^{1,2}$, Enzo Cazzanelli³,4, Angela Fasanella ${ }^{3}$, Marco Castriota3,5* \\ ${ }^{1}$ Donostia International Physics Center (DIPC), Donostia-San Sebastian, Spain \\ ${ }^{2}$ Centro de Física de Materiales (CSIC/UPV-EHU), Donostia-San Sebastian, Spain \\ ${ }^{3}$ Physics Department, University of Calabria, Cubo 31C, Rende (Cosenza), Italy \\ ${ }^{4}$ Notredame s.r.l., c/o Physics Department, University of Calabria, Rende, Italy \\ ${ }^{5} \mathrm{CNR}$, Istituto di Nanotecnologia NANOTEC, SS di Cosenza, Via P. Bucci Cubo 31/C, Rende (CS), Italy \\ Email: *marco.castriota@fis.unical.it
}

How to cite this paper: Mohammed, M.S.G., Cazzanelli, E., Fasanella, A. and Castriota, M. (2018) Silicon Nanocrystals on the Surface of Standard Si Wafers: A Micro-Raman Investigation. Journal of Materials Science and Chemical Engineering, 6, 104-116.

https://doi.org/10.4236/msce.2018.67012

Received: April 14, 2018

Accepted: July 15, 2018

Published: July 18, 2018

Copyright () 2018 by authors and Scientific Research Publishing Inc. This work is licensed under the Creative Commons Attribution International License (CC BY 4.0).

http://creativecommons.org/licenses/by/4.0/

\begin{abstract}
The presence of silicon nanocrystals on the surface of standard wafer samples of $\mathrm{Si}$, conserved under "usual" laboratory conditions, has been investigated by micro-Raman analysis, performed for increasing intensity of laser irradiation. The poor thermal connection of such small crystals to the Si wafer bulk allows for the appearance of two well distinct Raman bands in the spectra, with a different evolution for increasing irradiance levels: the first, expected, due to bulk silicon response, the other one assignable to the silicon nanocrystals. A careful analysis of peak position and linewidth has been carried out, both for the Raman contribution from the nanocrystals, reaching high temperatures under irradiation (up to $1400 \mathrm{~K}$ ), and for the one from the "bulk" Si, which remains practically at room temperature. The analysis of the spectra and the comparison with previous studies on nc-Si suggest that such nanocrystals do not have a very small size, so that the observed changes of spectral parameters are mainly due to laser heating, rather than quantum confinement effects. In any case, we performed also an independent size determination by AFM mapping, confirming a size distribution well peaked between 50 and $100 \mathrm{~nm}$. As a corollary from this analysis, we get the indication that apparent linewidths and positions, at low laser irradiation levels, can be slightly changed in the presence of nc-Si on the surface. It is due to the different thermal responses of bulk and nanocrystalline components, inducing unresolved separate components; this hypothesis suggests reanalysing some previous experimental data, in particular for many Raman spectra of Si collected at "room temperature".
\end{abstract}

\section{Keywords}

Silicon, Nanocrystals, Laser Heating, Raman Linewidth, AFM Measurement 


\section{Introduction}

Silicon has been one of the most studied elements since the great development of the electronic technology [1] [2], nevertheless it can still offer some surprising behaviours to the possible scientific investigations carried out on it. In particular, the physical properties of confined forms of silicon have been investigated in the recent years [3] [4] [5] [6], concerning in particular porous silicon [7] [8] and Si nanocrystals [9]-[17].

Among the several techniques to investigate such properties, Raman spectroscopy is quite useful, in particular in its recent micro-spectroscopic development. In fact, it is possible to excite very small regions of $\mathrm{Si}$ samples with concentrated laser power and monitor the thermal responses by analysing the collected Raman spectra.

In the present work, we report on several micro-Raman investigations performed on different standard Si wafers, which demonstrate the occurrence of $\mathrm{Si}$ nanocrystals at the surface of these wafers, having a quite different thermal response to the laser beam with respect to the bulk silicon. In conditions of strong laser irradiance these nanoparticles reach very high temperatures (estimated up to $\sim 1400 \mathrm{~K}$ ); while the bulk $\mathrm{Si}$, because of its high thermal conductivity, does not exceed appreciably room temperature. The behaviour of these nanocrystals under changing laser irradiation has been compared with the previously reported investigations about quantum confinement and localised heating phenomena, ascribed also to nanocrystals of different sizes, to better understand what kind of effect is prevalent in our case.

In addition, the effects of the nc-Si presence on the observed Raman spectral parameters have been investigated even for lower irradiance values, to explain some apparent changes in the experimentally observed lineshapes of the Raman spectrum of silicon wafers with respect to the values observed for the best bulk samples.

\section{Experimental}

\section{Samples}

The Si wafers studied in this work have been kindly provided by prof. Pavesi, of Physics department of Trento University, and by Dr. Toccoli, of the CNR-Center of Trento. In both cases the wafers were of commercial origin but well polished. With regard to the doping level, all the samples can be considered weakly $\mathrm{p}$ doped, so that the Raman lineshape of the principal one-phonon mode at $521 \mathrm{~cm}^{-1}$ is not appreciably different from the symmetric Lorentzian shape of the intrinsic material. We investigated regions of the Si samples appearing clean from corrosion or chemical transformation, at least at macrocopic level. Under microscopic examination, on the contrary, many dark spots are revealed: they can be due to the effect of common pollution agent present in the atmosphere of a laboratory. For aim of comparison with this "natural aging" damage, a sample was purposely damaged by producing scratching on its surface, and the 
scratched region has been analysed both by AFM and by micro-Raman spectroscopy: it shows about the same results as the "naturally" damaged spot of the previously studied samples.

\section{Characterization techniques}

An investigation was carried out by an AFM apparatus, on the best-polished surface of a wafer from CNR-Trento and on two scratched samples. The apparatus was working in a no contact mode with a tip MPP-11100-10 of n-doped Si $(\mathrm{Sb})$, having a resistivity of $0.01-0.023 \mathrm{ohm} \cdot \mathrm{cm}$ and a force constant of $40 \mathrm{~N} / \mathrm{m}$. $\mathrm{Z}$ resolution $2 \mathrm{~nm}$ and $\mathrm{XY}$ resolution $16 \mathrm{~nm}$.

Micro-Raman investigations are the main component of the present research. All of them have been performed by a Horiba Yobin-Yvon apparatus Labram, consisting of a spectrometer equipped with two gratings (either 1800 grooves $/ \mathrm{mm}$ or 600 grooves $/ \mathrm{mm}$ ) and a notch/edge filter, a cooled CCD detector of $1024 \times$ 256 pixels and an Olympus microscope interfaced to the spectrometer, with several objectives $(100 \times, 50 \times, 20 \times)$, for exploring the samples and for focusing the laser beam on them during the Raman measurements. As laser sources a He-Ne laser (633 nm line) of $17 \mathrm{~mW}$ power (about $6 \mathrm{~mW}$ measured on the sample) was used for most of the experiment, while some other ones, more recent, were also carried out by using a $532 \mathrm{~nm}$ line of a diode laser, with a maximum emitted power of $100 \mathrm{~mW}$.

\section{Results and Discussion}

\section{Heating of nanocrystals}

Two kinds of experiment have been basically performed to test the thermal response of the various silicon forms present on the surface of the wafers. The first one can be labelled as Z-scan, because the focus of the laser, with the maximum power (no filters), has been moved toward the sample by fixed amount, typically in steps of 0.5 or 1 micrometers, so that the irradiance on the surface of the impinged spot varies versus the distance from the objective, $Z$. This functional dependence can be approximated as $\sim 1 / Z^{2}$ when approaching the best focusing condition from greater distances, it exhibits a maximum for $\mathrm{Z}$ corresponding to the best focus and it decreases again when the focus "enters" into the sample. In the case of clean regions of the wafers, where only "bulk" Si was present, we just obtained a bell shaped curve for the Raman intensity of the principal peak of $\mathrm{Si}$ vs. $\mathrm{Z}$, with a maximum at the best focusing configuration. On the contrary, in several cases of "naturally" damaged regions we observed a quite complex and interesting evolution of the Raman spectra. Initially, for distances far away from the best focussing point (about 10 microns) the standard spectrum of bulk $\mathrm{Si}$ appeared, with the principal peak of $\mathrm{F}_{2 \mathrm{~g}}$ symmetry, its overtone just below $1000 \mathrm{~cm}^{-1}$ and the overtone of acoustic mode at $300 \mathrm{~cm}^{-1}$. The principal peak appears with a position $\omega \sim 520 \mathrm{~cm}^{-1}$ and a full width $\Gamma \sim 6 \mathrm{~cm}^{-1}$. On approaching the best focus condition, the width seems to increase until a splitting into two components become evident. Finally, under the highest laser 
irradiation at the right focal distance the lower frequency component strongly broadens and downshifts while the high frequency one maintains about the same initial values of $\omega$ and $\Gamma$. To be more precise, the position $\omega$ of the high frequency component shows a slight upshift while the measured $\Gamma$ values appear even smaller with respect to the initial spectra with poor focalization. Such evolution has been observed for many spots in different Si wafers, coming from different laboratories, by using the green $(532 \mathrm{~nm})$ laser focused by a $100 \times$ objective and also by using a full power He-Ne laser with the same objective; in this last case we estimate the irradiance corresponding to such experimental conditions to be in the range $0.5 \div 1 \mathrm{GW} / \mathrm{m}^{2}$. Some spectra representative of this evolution are shown in Figure 1, among many observed during a single $\mathrm{Z}$ scan. For lower irradiances at the $633 \mathrm{~nm}$ laser focus, obtained by using either filters or different objectives, the splitting effect was never observed; suggesting that a level of irradiance as reported above is just matching the threshold necessary for triggering the well observable effect. The other kind of measurement was a micro-Raman mapping, at fixed focal distance, of a region surrounding a point exhibiting the splitting. The change of the focus conditions due to the topography of the sample, combined with the changing density of nanocrystals, generate various spectral shapes, similar to those obtained for different $Z$ values.

The observation of two Raman bands of Si for laser powers above certain threshold values was previously reported in the work of Gibilisco et al. [15] where $\mathrm{Si}$ nanocrystals, purposely grown from a vapor phase on a graphite substrate, were impinged by a laser beam with powers of about $5 \mathrm{~mW}$, similar to our case. This similarity of the findings suggests that $\mathrm{Si}$ nanocrystals are present on the surface of our wafer samples, and their connection to the bulk do not allow a good thermal conduction, so that the heat induced by laser irradiation cannot be carried away. Therefore, we obtain very high local temperatures of nc-Si, revealed by the $\omega$ and $\Gamma$ values of the downshifted Raman peak, while the part of the sample with good thermal connection (the bulk) generates the higher frequency Raman band, with spectral parameters typical of room temperature. In principle, the temperature can be measured by the Stokes/anti-Stokes intensity ratio, but it was impossible in our case, where the temperature depends critically on the focusing, not perfectly reproducible during sequential Z-scans; moreover, our spectrometer did not allow a simultaneous measurement of Stokes and anti-Stokes frequency ranges. Therefore temperatures of bulk Si and $\mathrm{nc}$-Si have been estimated in another way, from the temperature dependence of Raman bands spectral parameters, peak position $\omega$ and linewidth $\Gamma$, reported in previous investigations on high temperature Raman spectra in bulk Si [18] [19]. Obviously, this approach is based on the assumption that anharmonic interactions in the analysed nc-Si, responsible for frequency and linewidth changes, are not appreciably different from those acting in the bulk Si. In particular, the measurements of by Balkanski et al. [18] are used to interpolate our data, because of their wide temperature range covered. For instance, in the bottom spectrum shown in 


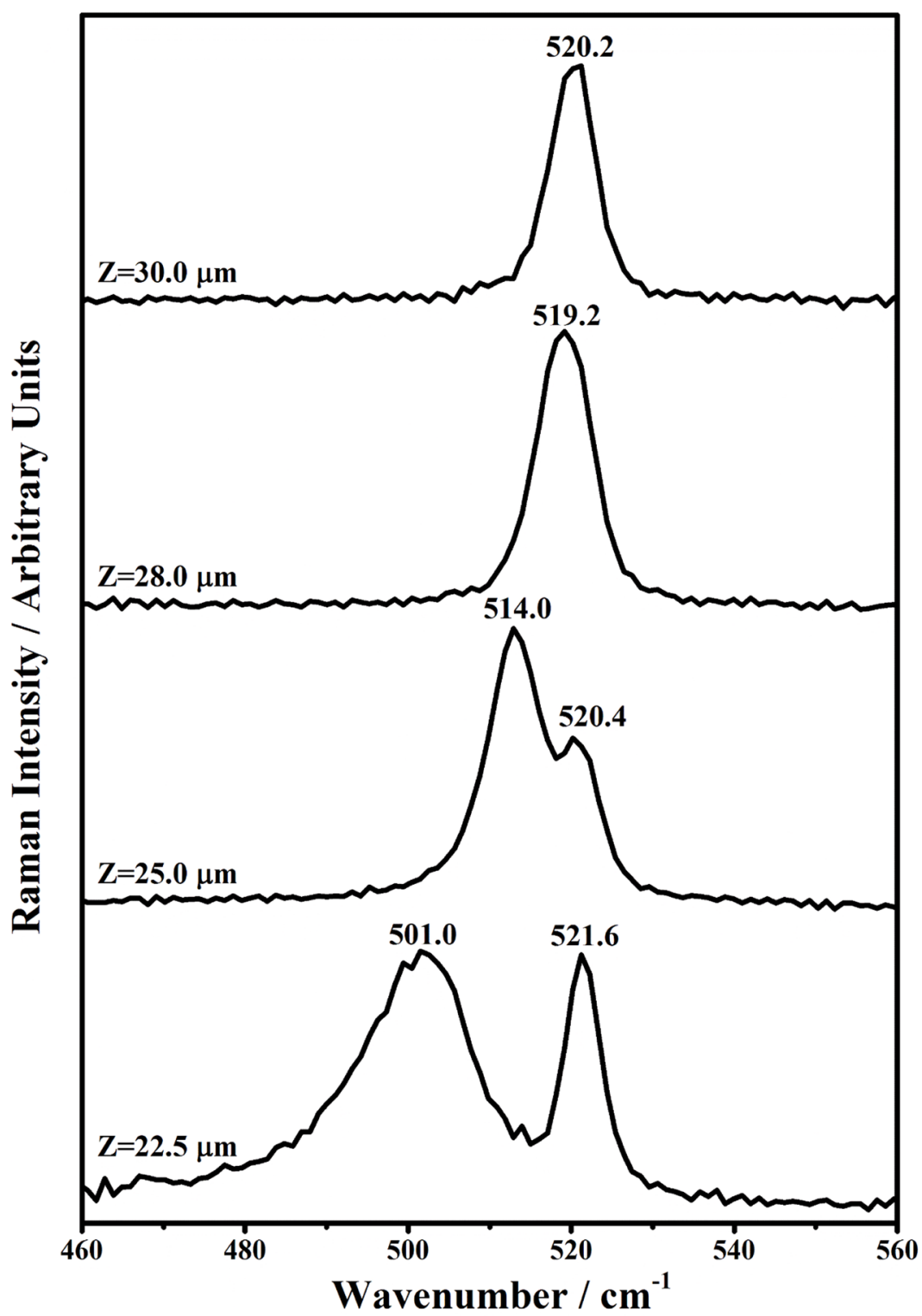

Figure 1. Four different Raman spectra are shown, collected during a Z-scan, with decreasing focal lengths from the top, reported within each figure, in $\mu \mathrm{m}$. The position $\omega$ and the full width $\Gamma$ of the peaks are calculated by a best fit procedure, based on Lorentzian functions, not shown in the figures for simplicity. In the $3^{\text {rd }}$ spectrum no fit is performed, because it is difficult and not very meaningful: only the apparent positions of the maxima are reported. The $633 \mathrm{~nm}$ laser was used, with full power, and a $100 \times$ objective.

Figure 1, the downshifted band allows two independent temperature estimates of the nanocrystals: the value obtained from $\Gamma, T(\Gamma)=1170 \mathrm{~K}$, and that derived from $\omega, \mathrm{T}(\omega)=1080 \mathrm{~K}$. These values of position and width were determined via a best fit procedure for each spectrum. In the Table 1 we report, for instance, the spectral parameters for both the Raman bands corresponding to some changing values of $Z$ during the same $Z$ scan, and the temperatures $T\left(\Gamma_{2}\right)$ and $T\left(\omega_{2}\right)$, estimated for the Si nanocrystals from the different values of width and position of 
Table 1. Spectral parameters $\omega$ and $\Gamma$ for the band 1 (bulk Si) and band 2 (nc-Si) corresponding to some different $\mathrm{Z}$ values of a single $\mathrm{Z}$ scan. The temperatures of nanocrystals are shown, in $\mathrm{K}$ degrees, independently estimated from peak position and width, by using a temperature dependences reported in literature (Balkanski, ref. 18). An objective 100x and the He-Ne laser with maximum power were used.

\begin{tabular}{ccccccc}
\hline$Z(\mu \mathrm{m})$ & $\omega_{2}\left(\mathrm{~cm}^{-1}\right)$ & $\Gamma_{2}\left(\mathrm{~cm}^{-1}\right)$ & $T\left(\omega_{2}\right) \pm 30 K(K)$ & $T\left(\Gamma_{2}\right) \pm 30 K(K)$ & $\omega_{1}\left(\mathrm{~cm}^{-1}\right)$ & $\Gamma_{1}\left(\mathrm{~cm}^{-1}\right)$ \\
\hline 23.5 & 507.5 & 10.5 & 860 & 830 & 521.4 & 4.5 \\
22.5 & 500.1 & 16.3 & 1080 & 1170 & 521.5 & 4.2 \\
19.5 & 495.7 & 20.0 & 1330 & 1360 & 521.9 & 3.7 \\
\hline
\end{tabular}

the low frequency band, respectively. This temperature evaluation, performed for many spectra, reveals, in general, small differences between $\mathrm{T}\left(\Gamma_{2}\right)$ and $\mathrm{T}\left(\omega_{2}\right)$, of the order of $10 \mathrm{~K}$, even for $\mathrm{T}>1000 \mathrm{~K}$. Obviously all the Raman spectra collected in this way derive from different nanocrystals distributed inside the radius of the focal spot and also constitute a time average during the collection time (few seconds) for each new focus configuration; it is assumed that a thermal equilibrium or quasi-equilibrium state can be reached within the measurement time. It can be expected, in principle, that an unknown distribution of temperatures among different nanocrystals determines the resulting Raman spectral shape. However, as starting approach, we adopt the strongly simplifying approximation of a single temperature for all the nanocrystals. It appears reasonable because of the quite symmetric shape of the Raman bands, allowing a very good fit with single Lorentzian function, and because of the relatively small difference between $\mathrm{T}\left(\Gamma_{2}\right)$ and $\mathrm{T}\left(\omega_{2}\right)$. In fact, independently from the width of the temperature distribution, we expect the peak position corresponding to the most frequent temperature to be very close to the observed maximum position of the total Raman band, resulting from the envelope of all the different nanocrystals contributions. On the other hand, in case of a broad temperature distribution for the Si nanocrystals, we expect an apparent width of the nc-Si total Raman band much greater than the width corresponding to the most frequent temperature; therefore, the $\mathrm{T}$ value derived from the width of the total Raman band would be expected much higher than the value derived from its maximum position. This discrepancy is not verified in remarkable way, and this fact can be ascribed to a relatively small temperature spread of the hot nanocrystals, so that only a small error arises by assuming for all of them an effective mean temperature. For all the measurements made with $633 \mathrm{~nm}$ laser (low power) and even for most of the ones performed with $532 \mathrm{~nm}$ laser (higher power) this assumption seems to be justified. The temperatures of the nanocrystals have been evaluated for many analysed spectra, within the above said approximation, finding values up to $\sim 1400 \mathrm{~K}$, in reasonable agreement with the data reported in a previous study on $\mathrm{nc}-\mathrm{Si}$, concerning similar effects, and based on Stokes/antiStokes method for temperature evaluation [20]. Only few experiments made with high laser power of $532 \mathrm{~nm}$ wavelength seem clearly to generate a multimodal temperature dis- 
tribution among the nc-Si component of the irradiated region, as suggested by the observation of several downshifted Raman bands.

The intensity ratio between the downshifted band, due to the hot Si nanocrystals, and the other one, coming from room temperature bulk $\mathrm{Si}$, is found strongly variable, depending on the samples and on the position. Among our measurements, we have found cases with very high relative intensity of nc-Si Raman band, more than in previously investigated similar systems [11]-[17]. For instance in another Z-scan we have found at the best focus position an intensity ratio of about 23 between the two bands, as it can be seen in Figure 2. These findings can further support the hypothesis of an enhancement effect for Raman scattering associated to Si-nanocrystals, as reported by different groups [21] [22].

\section{Size determination of $\mathrm{nc}-\mathrm{Si}$}

The observed spectral evolution suggests that nc-Si subjected to the selective heating under laser irradiation do not show an appreciable evidence of quantum confinement effects, behaving substantially like the bulk $\mathrm{Si}$, because they do not exhibit appreciable downshifts and broadenings [14] [23] [24] [25] at room T (low irradiances); they just have a poor thermal connection to the bulk. Therefore, it is reasonable to guess an average value for their size not very small, which confirms the validity of the assumption about the anarmonicity of these nanocrystals not different from that in bulk Si.

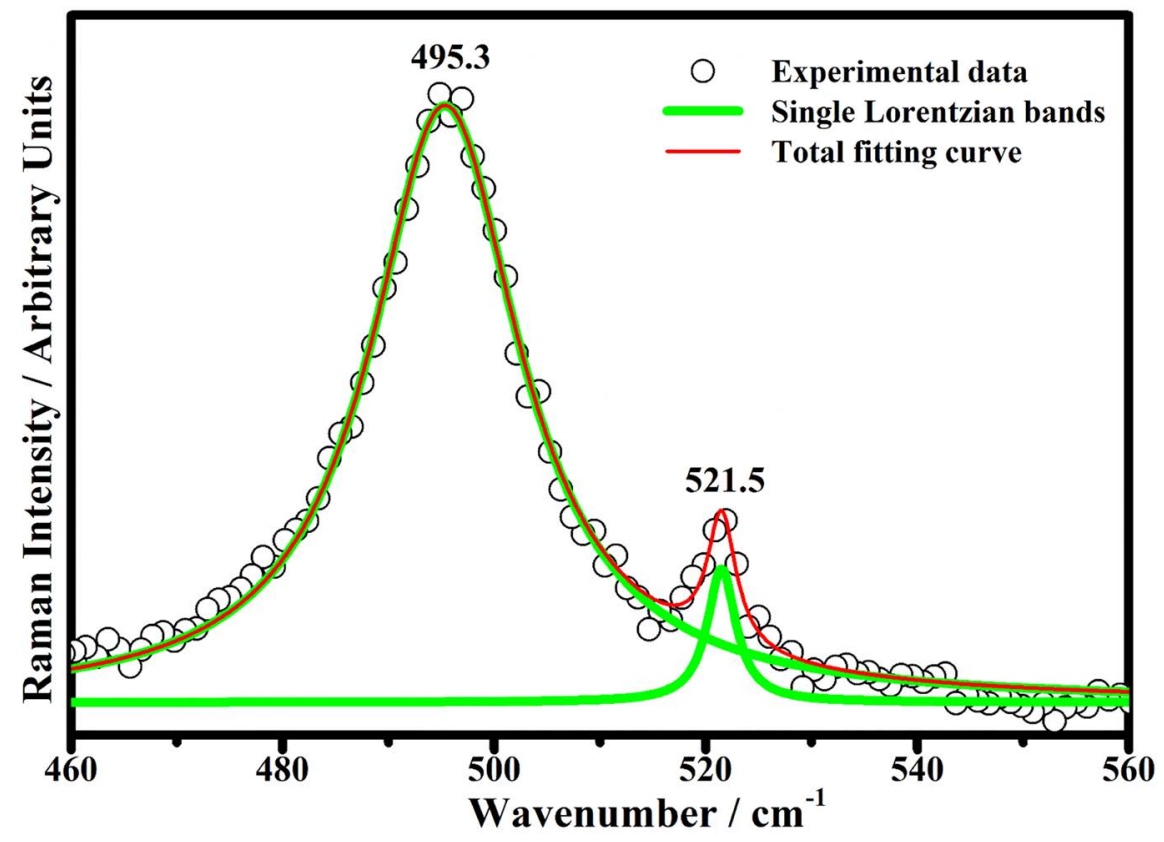

Figure 2. Raman spectrum from a Z-scan, exhibiting the maximum intensity ratio between the nc-Si component, reaching an estimated temperature of $1230 \mathrm{~K}$, and the bulk Si component, revealing a constant room temperature. Peak positions, widths and intensities are calculated by a fitting procedure, as in Figure 1, and the fitting Lorentzian functions are shown together with their sum (cumulative fit). The $633 \mathrm{~nm}$ laser was used, with full power, and a $100 \times$ objective. 
A direct measurement of the size was performed by an AFM apparatus, on a scratch line due to aging, which was associated to a Raman band splitting similar to the one shown in Figure 1. The optical image, collected by the $100 \times$ objective, and the corresponding AFM map are reported in Figure 3. By analysing this AFM map, we obtain the histogram of size distribution of the Si nanocrystals, reported in Figure 4. Most of them are in the range $50-100 \mathrm{~nm}$. On the basis of previous studies of the temperature dependent Raman spectra of nc-Si of different size [13] [14] [17] [24] we can expect that they have about the same temperature dependence of Raman spectral parameters as that reported by Balkanski [18] for bulk Si, when strongly heated. It confirms that our method for temperature evaluation was quite reasonable. Moreover, their size distribution rather narrow is consistent with the adopted single temperature approximation. Finally, this fact explains why they do not show the typical asymmetry, downshifts
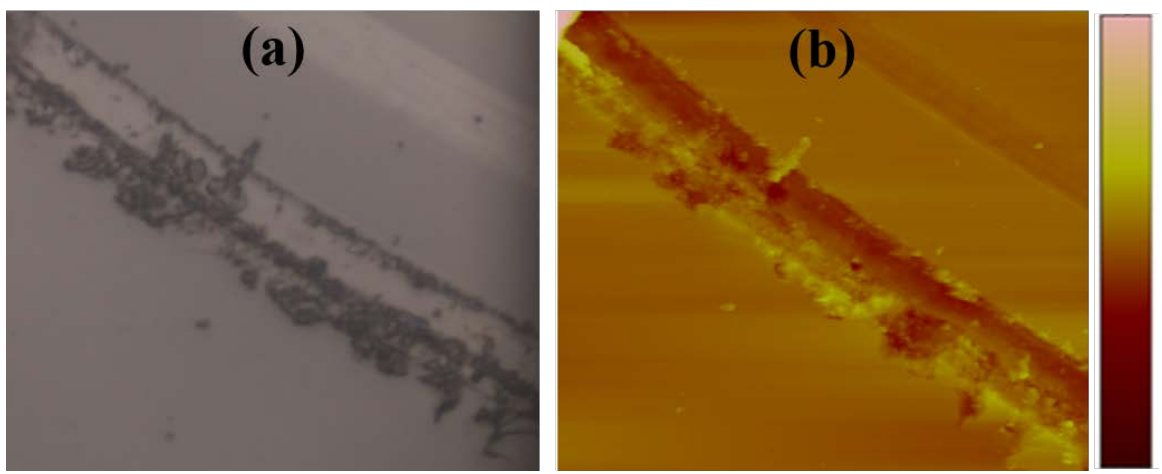

Figure 3. Region with Silicon nanocrystals (a) Image collected by optical microscope, with objective 100×; (b) AFM mapping, represented in a false colours scale.

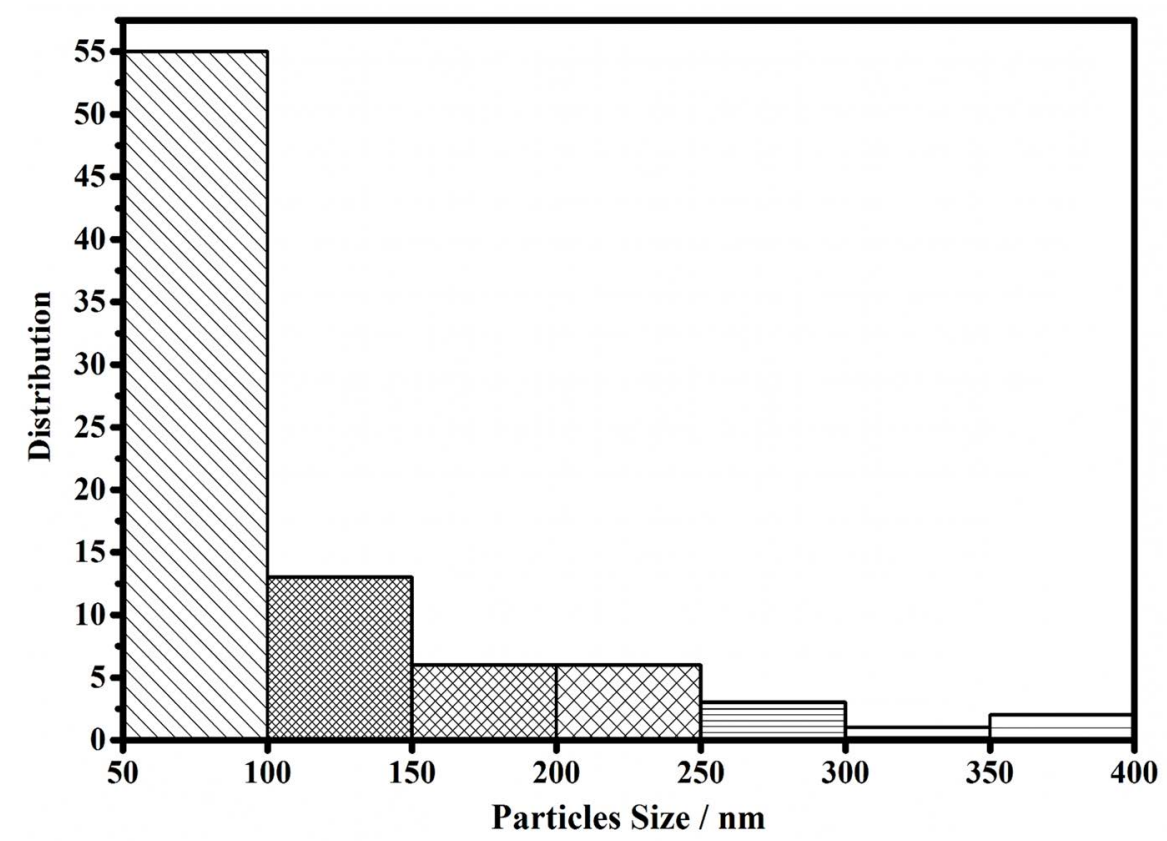

Figure 4. Size distribution histogram of the nanocrystals present in Figure 3, as measured by AFM. 
or broadenings related to quantum confinement, observed for smaller nanocrystals [25] [26], because in the literature there is a general agreement that quantum confinement is not relevant for particles greater than $50 \mathrm{~nm}$, as in our case.

\section{Apparent linewidths for low irradiance}

The evidences reported above show that the "natural" aging process in standard Si wafer samples can generate on the surface a population of nanocrystals, with the size distribution peaked between 50 and $100 \mathrm{~nm}$; they exhibit a remarkable apparent splitting of Raman band $\mathrm{F}_{2 \mathrm{~g}}$ when subjected to a laser irradiance above a threshold of about $0.5 \mathrm{GW} / \mathrm{m}^{2}$, in agreement with the data of Nikolenko [23]. However, as previously mentioned, an appreciable occurrence of nc-Si can slightly modify the Raman spectral parameters even in low irradiance conditions. In fact the apparent linewidth $\Gamma$ of $F_{2 g}$ mode, for the initial defocused positions of Z-scan (lower irradiance), appears greater than the usual $\Gamma$ values given by the literature for bulk Si: values of about $6 \mathrm{~cm}^{-1}$ are observed, against about $4 \mathrm{~cm}^{-1}$, respectively. On the contrary, when approaching the best focus condition, the stronger irradiance of the laser induces a remarkable downshift and broadening of the nc-Si Raman band, while the other one, assigned to the bulk $\mathrm{Si}$, seems to exhibit a little upshift $\left(\sim 1.5 \mathrm{~cm}^{-1}\right)$ and narrowing (from 6 to less than $4 \mathrm{~cm}^{-1}$ ), with respect to the lower irradiance initial conditions (see Figure 1 top and bottom and also the spectral parameters for bulk Si band in Table 1). Obviously, a "cooling effect" of the high intensity laser beam on the bulk $\mathrm{Si}$ is impossible, out of discussion; in any case this reported change is significant and deserves at least some tentative explanation. A reasonable hypothesis which can describe the observed evolution of bulk Si band is that, in defocused condition, in the regions of the samples where an appreciable nc-Si fraction exist, the observed Raman peak is already a convolution, unresolved, of bulk $\mathrm{Si}$ and nc-Si contributions, having two slightly different values of $\omega$ and $\Gamma$. Our spectrometer is not able to resolve, at room $\mathrm{T}$, these two components when they are too close; therefore, to help the explanation of our qualitative hypothesis, we show in Figure 5 a possible simulation of the observed Raman band, obtained by postulating two peaks with spectral parameters not far from the usually measured ones: the bulk Si peak with $\omega_{1}=521.5 \mathrm{~cm}^{-1}$ and $\Gamma_{1}=3.6 \mathrm{~cm}^{-1}$ and the nc-Si peak with $\omega_{2}=518.5 \mathrm{~cm}^{-1}$ and $\Gamma_{2}=4.2 \mathrm{~cm}^{-1}$, due to a slightly higher temperature; we also assign the same intensity (area under peak) to these components, for simplicity. The resulting convolution is a curve which can be still best fitted by a Lorentzian function, with apparent peak position $\omega=520.2 \mathrm{~cm}^{-1}$ and width $\Gamma=6 \mathrm{~cm}^{-1}$, which are very close to the commonly observed initial values in our Z scan far from the best focus (see, for instance, Figure 1, top spectrum).

An alternative explanation of the observed excess of Raman linewidth in our samples at low irradiance can be proposed in terms of weak quantum confinement effects due to very small nanocrystals, but it contrasts with the direct AFM measurement of the nanocrystals size distribution, showing the absence of any component below $50 \mathrm{~nm}$. 


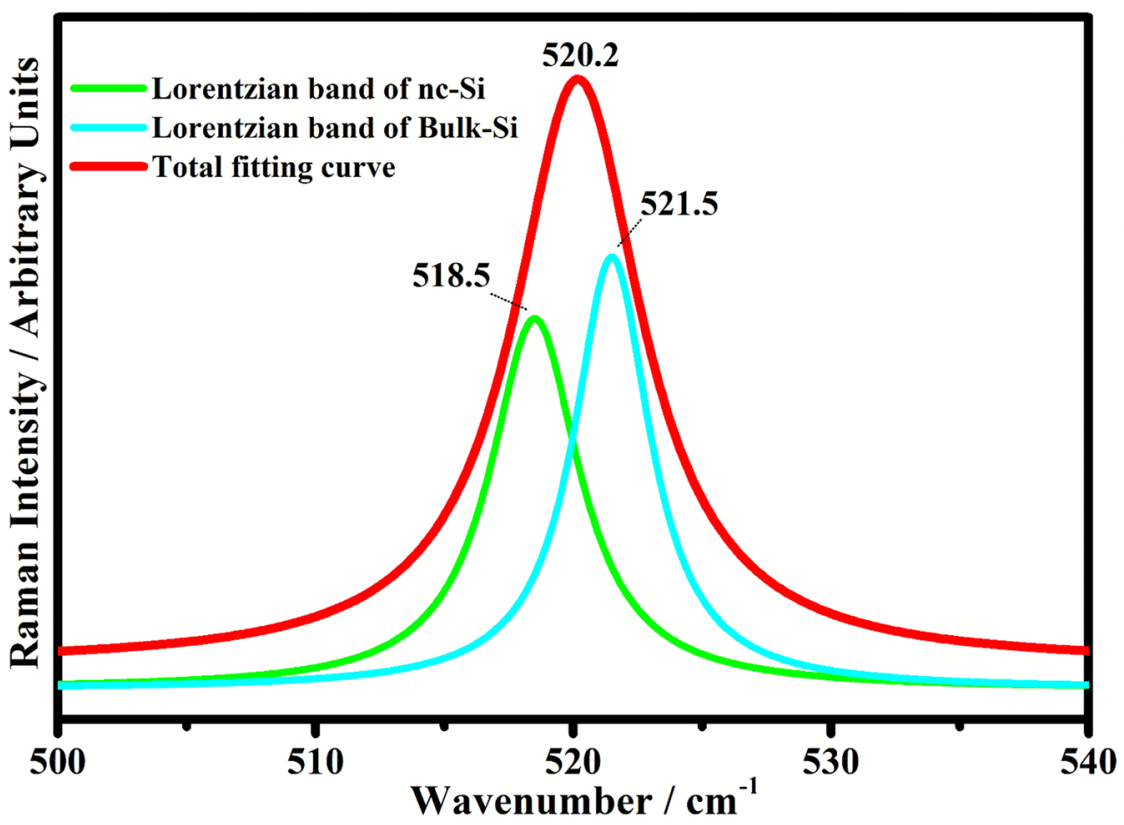

Figure 5. Simulation of a possible Raman band of Silicon, at low laser irradiance, based on the hypothesis of two contributions. A bulk Si peak at room temperature, represented by a Lorentzian curve with position $\omega_{1}=521.5 \mathrm{~cm}^{-1}$, and full width $\Gamma_{1}=3.6 \mathrm{~cm}^{-1}$, and another one, generated by nc-Si reaching a slightly higher temperature, represented by a second Lorentzian function, with $\omega_{2}=518.5 \mathrm{~cm}^{-1}$ and $\Gamma_{2}=4.2 \mathrm{~cm}^{-1}$. The area below these two Lorentzians is assumed to be equal, for simplicity. The sum of these two contribution is best fitted by a third Lorentzian, with $\omega=520.2 \mathrm{~cm}^{-1}$ and $\Gamma=6 \mathrm{~cm}^{-1}$, values quite similar to those of unique apparent peaks at low irradiance.

Finally, another possible explanation of the apparent narrowing of bulk Raman band for the highest irradiance at the best focus condition, could be proposed in terms of an instrumental effect, i.e. better spectral resolution for the best focus condition. To check that hypothesis a Z-scan test on a clean bulk Si sample was performed, at low laser power: by moving the objective along $\mathrm{Z}$ for $7.5 \mu \mathrm{m}$ (similar distance value to that of Figure 1 between top and bottom) we observe for all the $\mathrm{Z}$ values the same width and position values for the $\mathrm{F}_{2 \mathrm{~g}}$ peak, consistent also with the usual data from literature, within the error bars given by the fitting procedure. This experimental test demonstrates that the small broadening observed for the principal Raman peak of $\mathrm{Si}$ is a real physical effect, due to an additional unresolved contribution associated to the nanocrystals presence in the "damaged" regions of our wafer samples.

\section{Conclusions}

In summary, we can state that standard silicon wafers, kept in normal conditions of conservation, can develop at the surface some amounts of nanocrystals, with sizes greater than $50 \mathrm{~nm}$, having a very poor thermal connection with the bulk, however not inducing quantum confinement effects. Under proper laser irradiation these nanoparticles reach very high temperatures (up to $\sim 1400 \mathrm{~K}$ ), while the bulk component exhibits a very small heating, because of its high thermal con- 
ductivity. The mean temperature of nc-Si can be estimated by using literature data about temperature dependence for the position and linewidth of the principal Raman peak $\mathrm{F}_{2 \mathrm{~g}}$ of bulk silicon.

This different thermal response between bulk and nc-Si is also responsible of apparent broadening of the Si Raman peak even in condition of low irradiance, when the strong splitting of the Raman bands is not observed. Therefore we suggest that some of the variance observed for the linewidth of Si Raman peak at room temperature can be ascribed to the presence of nanocrystals developed on the surface of Si samples in standard laboratory conditions of storage.

\section{Acknowledgements}

This work was partially supported by POR CALABRIA FESR-FSE 2014-2020-ASSE I-PROMOZIONE DELLA RICERCA E DELL'INNOVAZIONE Obiettivo specifico 1.2 Azione 1.2.2 Project: "MERAVIGLIE".

The authors would like to thank prof. Lorenzo Pavesi from University of Trento, Dr. Tullio Toccoli and Dr. Nicola Coppedè from CNR-FBK of Trento and Dr. Maria Penelope De Santo from University of Calabria for kindly providing different Si samples, used in this work, and for some useful discussion. Moreover, we thank Tiziana Ritacco and Dr. Michele Giocondo from IMIP-CNR, Nanotec group of Calabria, for help in AFM measurements.

\section{References}

[1] Basu, S. (ed.) (2011) Crystalline Silicon-Properties and Uses. Intech. https://doi.org/10.5772/844

[2] Kissinger, G. and Pizzini, S. (eds.) (2015) Silicon, Germanium and Their Alloys: Growth, Defects, Impurities and Nanocrystals. CRC Press, Taylor and Francis Group, London.

[3] Ossicini, S., Pavesi, L. and Priolo, F. (eds.) (2003) Light Emitting Silicon for Microphotonics. Springer Tracts in Modern Physics 194, Springer, Berlin Heidelberg. https://doi.org/10.1007/b13588

[4] Theodorakos, I., Zergioti, I., Vamvakas, V., Tsoukalas, D. and Raptis, Y.S. (2014) Picosecond and Nanosecond Laser Annealing and Simulation of Amorphous Silicon Thin Films for Solar Cell Applications. Journal of Applied Physics, 115, 043108. https://doi.org/10.1063/1.4863402

[5] Deschaines, T., Hodkiewicz, J. and Henson, P. (2009) Characterization of Amorphous and Microcrystalline Silicon Using Raman Spectroscopy. Thermo Fisher Scientific, Madison, WI.

[6] Piscanec, S., Cantoro, M., Ferrari, A.C., Zapien, J.A., Lifshitz, Y., Lee, S.T., Hofmann, S. and Robertson, J. (2003) Raman Spectroscopy of Silicon Nanowires. Physical Review B, 68, 241312. https://doi.org/10.1103/PhysRevB.68.241312

[7] Canham, L. (ed.) (1997) Properties of Porous Silicon. INSPEC, The Institution of Electrical Engineers, London.

[8] Moreno, J.D., Montoya, E. and Martı, J.M. (1998) Influence of Wavelength on the Raman Line Shape in Porous Silicon. Journal of Applied Physics, 84, 2349.

[9] Pavesi, L. and Turan, R. (eds.) (2010) Silicon Nanocrystals, Fundamentals, Synthesis 
and Applications, Wiley-VCH Verlag, Weinheim. https://doi.org/10.1002/9783527629954

[10] Heitmann, J., Mueller, F., Zacharias, M. and Goesele, U. (2005) Silicon Nanocrystals: Size Matters. Advanced Materials, 17, 795. https://doi.org/10.1002/adma.200401126

[11] Bustarret, E., Hachicha, M.A. and Brunel, M. (1988) Experimental Determination of the Nanocrystalline Volume Fraction in Silicon Thin Films from Raman Spectroscopy. Applied Physics Letters, 52, 1675. https://doi.org/10.1063/1.99054

[12] Faraci, G., Gibilisco, S., Russo, P., Pennisi, A.R., Compagnini, G., Battiato, S., Puglisi, R. and La Rosa, S. (2005) $\mathrm{Si} / \mathrm{SiO}_{2}$ Core Shell Clusters Probed by Raman Spectroscopy. The European Physical Journal B, 46, 457. https://doi.org/10.1140/epjb/e2005-00274-4

[13] Faraci, G., Gibilisco, S., Russo, P. and Pennisi, A.R. (2006) Modified Raman Confinement Model for Si Nanocrystals. Physical Review B, 73, 033307. https://doi.org/10.1103/PhysRevB.73.033307

[14] Faraci, G., Gibilisco, S. and Pennisi, A.R. (2009) Quantum Confinement and Thermal Effects on the Raman Spectra of Si Nanocrystals. Physical Review B, 80,193410. https://doi.org/10.1103/PhysRevB.80.193410

[15] Gibilisco, S., Faraci, G., Pennisi, A.R. and Irrera, A. (2012) Laser Induced Heating of Si Nanocrystals. Journal of Non-Crystalline Solids, 356, 1948. https://doi.org/10.1016/j.jnoncrysol.2010.05.035

[16] Hessel, C.M., Wei, J., Reid, D., Fujii, H., Downer, M.C. and Korgel, B.A. (2012) Raman Spectroscopy of Oxide-Embedded and Ligand-Stabilized Silicon Nanocrystals. The Journal of Physical Chemistry Letters, 3, 1089-1093. https://doi.org/10.1021/jz300309n

[17] Han, L., Zeman, M. and Smets, A.H.M. (2015) Raman Study of Laser-Induced Heating Effects in Free-Standing Silicon Nanocrystals. Nanoscale, 7, 8389-8397. https://doi.org/10.1039/C5NR00468C

[18] Balkanski, M., Wallis, R.F. and Haro, E. (1983) Anharmonic Effects in Light Scattering Due to Optical Phonons in Silicon. Physical Review B, 28, 1928-1934. https://doi.org/10.1103/PhysRevB.28.1928

[19] Tsu, R. and Hernandez, J.G. (1982) Temperature Dependence of Silicon Raman Lines. Applied Physics Letters, 41, 1016-1018. https://doi.org/10.1063/1.93394

[20] Faraci, G., Gibilisco, S. and Pennisi, A.R. (2009) Superheating of Silicon Nanocrystals Observed by Raman Spectroscopy. Physics Letters A, 373, 3779-3782.

[21] Mannino, G., Alberti, A., Ruggeri, R., Libertino, S., Pennisi, A.R. and Faraci, G. (2015) Octahedral Faceted Si Nanoparticles as Optical Traps with Enormous Yield Amplification. Nature Scientific Reports, 5, Article No. 8354. https://doi.org/10.1038/srep08354

[22] Sirleto, L., Ferrara, M.A., Nikitin, T., Novikov, S. and Khriachtchev, L. (2012) Giant Raman Gain in Silicon Nanocrystals. Nature Communications, 3, Article No. 1220.

[23] Nicolenko, A.S. (2013) Temperature Dependence of Raman Spectra of Silicon Nanocrystals in Oxide Matrix. Ukrainian Journal of Physics, 58, 980-987.

[24] Duan, Y., Kong, J.F. and Shen, W.Z. (2012) Raman Investigation of Silicon Nanocrystals: Quantum Confinement and Laser-Induced Thermal Effects. Journal of Raman Spectroscopy, 43, 756-760.

[25] Ke, W., Feng, X. and Huang, Y. (2011) The Effect of Si-Nanocrystal Size Distribution on Raman Spectrum. Journal of Applied Physics, 109, Article ID: 083526. https://doi.org/10.1063/1.3569888 
[26] Gupta, S.K. and Jha, P.K. (2009) Modified Phonon Confinement Model for Size Dependent Raman Shift and Linewidth of Silicon Nanocrystals. Solid State Communications, 149, 1989. 